\title{
Safety of phosphatidylserine containing omega-3 fatty acids in non-demented elderly: a double- blind placebo-controlled trial followed by an open-label extension
}

\author{
Veronika Vakhapova', Yael Richter ${ }^{2}$, Tzafra Cohen², Yael Herzog ${ }^{2}$ and Amos D Korczyn ${ }^{3^{*}}$
}

\begin{abstract}
Background: Phosphatidylserine (PS) is a naturally occurring phospholipid present in the inner leaflet of mammalian plasma membranes. Administration of PS extracted from bovine cortex (BC-PS), which contains high levels of omega-3 long chain polyunsaturated fatty acid (LC-PUFA) attached to its backbone, resulted in positive effects on brain functions such as learning and memory. Recently, a novel marine-sourced PS with omega-3 LCPUFA attached to its backbone was developed (PS-DHA). In the present study, we evaluated the safety profile of the novel PS preparation in non-demented elderly with memory complaints. The efficacy study of this novel formulation indicated that PS-DHA may ameliorate cognitive deficits in non-demented elderly population.
\end{abstract}

Methods: 157 non-demented elderly participants with memory complaints were randomized to receive either PS-DHA (300 mg PS/day) or placebo for 15 weeks. Standard biochemical and hematological safety parameters, blood pressure and heart rate were evaluated at baseline and endpoint. 122 participants continued into an open-label extension for additional 15 weeks, in which they all consumed PS-DHA (100 mg PS/day) and were evaluated for their blood pressure, heart rate and weight at endpoint. Adverse events were monitored throughout the double-blind and open-label phases.

Results: 131 participants completed the double-blind phase. No significant differences were found in any of the tested safety parameters between the study groups, or within each group. 121 participants completed the openlabel phase. At the end of this phase, there was a reduction in resting diastolic blood pressure and a slight weight gain among participants who consumed PS-DHA for 30 weeks.

Conclusions: The results of this study indicate that consumption of PS-DHA at a dosage of $300 \mathrm{mg}$ PS/day for 15 weeks, or $100 \mathrm{mg}$ PS/day for 30 weeks, is safe, well tolerated, and does not produce any negative effects in the tested parameters.

Trial registration: clinicaltrials. gov, identifier: NCT00437983

\section{Background}

Phosphatidylserine (PS) is a naturally occurring phospholipid present in the inner leaflet of mammalian plasma membranes. In humans, PS is most concentrated in the brain where it comprises $15 \%$ of the total phospholipid pool. PS has been shown to play a key role in the functioning of neuron membranes, such as signal

\footnotetext{
* Correspondence: amoskor@post.tau.ac.il

${ }^{3}$ Sieratzki Chair of Neurology, Tel-Aviv University Medical School, Ramat Aviv, Israel
}

Full list of author information is available at the end of the article transduction, secretory vesicle release and cell-to-cell communication [1].

The administration of PS extracted from bovine cortex (BC-PS) has positive effects on brain function. BC-PS was shown to improve learning and memory in age-associated memory impaired subjects [2], to enhance behavioral and cognitive parameters in geriatric patients [3], and to improve cognitive performance of Alzheimer's disease (AD) patients $[4,5]$. Although the primary objective of clinical studies involving BC-PS was to test efficacy, no significant adverse events were reported following oral

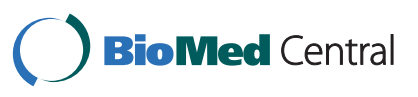


administration of BC-PS at doses of 300-400 mg/day for up to 3 months [2-8]. In the largest double-blind, placebo-controlled trial [3], comprising 494 participants, only one subject dropped out because of an adverse event, as compared to seven drop-outs from the placebo group.

Due to safety concerns about potential contamination by bovine spongiform encephalopathy (BSE) prions in recent years, alternatives to $\mathrm{BC}$-PS, such as soy derived PS (SB-PS), have been developed. SB-PS however, differs considerably in its fatty acid composition as compared to mammalian brain PS, and while SB-PS was shown to attenuate both physical [9] and mental stress [10,11], further research is required to determine its ability to promote cognitive functioning $[12,13]$. The safety of SBPS was tested in a double-blind placebo controlled study on 120 elderly [14]. Hematological and biochemical parameters along with vital signs and adverse events were evaluated after 6 and 12 weeks of treatment. The study conclusion was that SB-PS is safe for administration to older persons if taken up to a dosage of $600 \mathrm{mg} /$ day [14].

To gain the benefits of mammalian PS without the attendant risks, a safe, marine-sourced PS with omega-3 long chain polyunsaturated fatty acid (LC-PUFA) attached to its backbone was developed. This compound was recently found to improve the symptoms of children with impaired visual sustained attention [15] and to protect middle-aged rats from scopolamine-induced deleterious effects [16]. However, the biochemical and hematological tolerability of PS with omega-3 attached to its backbone have not been presented until now.

In the present report we describe the safety of a novel formulation of PS with omega-3 LC-PUFA, mainly docosahexaenoic acid (DHA), attached to its glycerol backbone (PS-DHA), in non-demented elderly. The relevant safety parameters were derived from biochemical and hematological variables, examination of vital signs and adverse events monitoring during the 15 weeks doubleblind placebo controlled study and from examination of vital signs and adverse events monitoring during the 15 additional weeks of the open-label extension phase. The efficacy of this novel formulation has also been tested in this population and the results of the double-blind phase are reported elsewhere [17]. Briefly, the study results indicated that PS-DHA may improve cognitive performance in non-demented elderly with memory complaints as PS-DHA administration resulted in an improvement of verbal immediate memory and in higher responders rates in comparison with the placebo group [17].

\section{Methods}

\section{Subjects}

Participants were recruited through advertisements in senior citizens homes, hospitals, and newspapers.
Approximately 700 elderly were screened for enrollment to the study. Out of them 157 non-demented participants with memory complaints met the previously described inclusion criteria [17]. Briefly, eligible participants were non-demented men or women between the ages of 50 and 90 years, with complaints of memory loss [18] and no evidence of a condition that could produce cognitive deterioration including AD, Parkinson's disease, stroke, normal pressure hydrocephalus, and other brain lesions including tumors, renal, respiratory, cardiac, and hepatic disease, diabetes mellitus, endocrine, metabolic or hematological disturbances unless well controlled, and malignancy not in remission for more than two years. Concomitant use of drugs or supplements affecting cognitive function was prohibited.

The study was conducted according to the principles of the Declaration of Helsinki and good clinical practice. The protocol was approved by the Ethics Committee of the Sourasky Medical Center, Tel-Aviv, Israel, and all volunteers gave written informed consent prior to participation.

\section{Study Design}

The study was designed as a single-center, randomized, double-blind, placebo-controlled, 15 weeks study, followed by an open-label extension of additional 15 weeks. At the first, double-blind, phase, participants were randomized according to a computerized process based on 6 and 8 blocks, in a 1:1 ratio stratified by gender, to receive three capsules per day of PS-DHA or a matched identically looking placebo (cellulose). The daily PS-DHA dosage provided $300 \mathrm{mg}$ PS and $79 \mathrm{mg}$ DHA+EPA (DHA:EPA ratio of 3:1). During the second, open-label, phase, participants consumed one capsule a day of PS-DHA. The daily dosage provided $100 \mathrm{mg}$ PS and $26 \mathrm{mg}$ DHA+EPA. PS-DHA (Vayacog ${ }^{\mathrm{TM}}$ ) was supplied by Enzymotec, Migdal HaEmeq, Israel.

Safety was evaluated by clinical laboratory assessments including biochemical and hematological parameters at baseline and endpoint of the double-blind phase and by adverse events recording, physical examination and measurement of vital signs and weight at baseline, week 7 and endpoint (week 15) of the double-blind phase and at the end of the open-label extension (week 30).

Blood samples were analyzed by the American Medical Laboratories (AML), Herzliya, Israel. Adverse events were monitored and recorded at each visit and by telephone contact every other week.

\section{Laboratory Parameters}

Biochemical parameters consisted of potassium, sodium, calcium, phosphorus, chloride, glucose, creatinine, blood urea nitrogen (BUN), bilirubin, total protein and lipid profile (total cholesterol, triglycerides, HDL, and LDL), 
alanine-aminotransferase (ALT), aspartateaminotransferase (AST), and alkaline phosphatase. Hematology consisted of red blood cell count, hematocrit, hemoglobin, white blood cell count and differential, platelets, MCV, $\mathrm{MCH}$, and $\mathrm{MCHC}$.

\section{Physical parameters}

The parameters assessed were weight, resting systolic and diastolic blood pressure, and pulse rate.

\section{Statistical Analysis}

Two-sided Student's t-test for dependent samples was used to analyze changes between different points in time in the tested parameters, in the whole group and in each gender separately, for both arms.

Two-sided t-test for independent samples was used to analyze differences between arms in the change between baseline and week 15 in blood parameters, vital signs and weight, in the whole group and in each gender separately and to detect any difference between groups in the frequency of adverse events.

Pearson's chi-square test for categorical variables was used to analyze the differences between groups in the number of participants who reported adverse events.

In the analysis of differences between and within groups, $\mathrm{P}$ values were adjusted for the number of parameters analyzed using Bonferroni correction. SAS statistical package (version 9.1) was used for all analyses.

\section{Results}

\section{Study Population}

A total of 157 participants underwent randomization (79 were assigned to PS-DHA treatment and 78 to placebo treatment). One hundred and thirty one participants completed the double-blind study (66 in the treatment group and 65 in the placebo group). Drop-outs were distributed equally over the two arms and reasons for discontinuation were generally similar across the treatment groups (Table 1). Average age of participants who completed the double-blind study $( \pm$ SD) was $72.42 \pm$ 8.02 in the PS-DHA group and $72.73 \pm 8.25$ in the placebo group. There were no significant differences between treatment groups in the incidence of existing disorders including cardiovascular disease and endocrine or metabolic disorders.

Sixty one participants from the PS-DHA group (PSDHA continuers) and 61 from the placebo group (PSnaive) continued into the open-label 15 weeks extension period. One participant from the PS-DHA naive group dropped out from the study due to protocol violation. Average age of participants who completed the openlabel extension $( \pm$ SD) was $72.36 \pm 7.93$ in the PS-DHA continuers group and $72.73 \pm 8.31$ in the PS-DHA naive group.
Table 1 Reasons for study discontinuation

\begin{tabular}{lll}
\hline Reason & PS-DHA & Placebo \\
\hline Protocol violation & 2 & 1 \\
\hline Withdrawn consent & 5 & 6 \\
\hline Adverse events* & 5 & 5 \\
\hline Severe adverse events** & $1^{\#}$ & $1^{\# \#}$ \\
\hline Sum & 13 & 13 \\
\hline
\end{tabular}

* Adverse events are specified in Table 6

** Classified by the study physician as not related to the study treatment

\# Hospitalization due to hyponatremia

\#\# Hospitalization due to atrial fibrillation and epigastric pain

\section{Safety parameters}

Physical parameters values during the double-blind phase are presented in Table 2. No significant changes from baseline in resting systolic and diastolic pressure, resting pulse rate and weight were found between treatment groups. A statistically significant increase in weight $(0.45 \pm$ $0.04 \mathrm{~kg}$ ) was detected in the PS-DHA group during the course of the double-blind phase. However, this elevation did not survive Bonferroni correction. Physical parameter values of PS-DHA continuers, who completed the openlabel phase, are presented in Table 3. A statistically significant decrease in resting diastolic BP $(3.1 \pm 0.3 \mathrm{mmHg})$ and a slight increase in weight $(0.61 \pm 0.05 \mathrm{~kg})$ were observed following 30 weeks of PS-DHA administration.

Among PS-DHA naive participants, who received PSDHA for 15 weeks, there were no significant changes in any of the tested physical parameters at the end of the open-label phase (data not shown).

Biochemical parameters values during the double-blind phase are presented in Table 4. No significant differences between groups were observed in the biochemical parameters, except for minor differences in sodium, calcium, chloride and females triglyceride levels. However, these differences did not survive Bonferroni correction and hence were rendered insignificant. In addition, during the course of the double-blind phase there were few parameters that showed a statistically significant change from baseline in the PS-DHA group, and other parameters in the placebo group. However again, the correction rendered them insignificant.

Hematological parameters values during the doubleblind phase are presented in Table 5 . No significant differences between groups were observed, except for a minor difference in the neutrophils count of the female population. Again, this difference did not survive Bonferroni correction and hence was rendered insignificant. In addition, during the course of the double-blind phase there were few hematological parameters that showed slight changes from baseline in the PS-DHA group, and other parameters in the placebo group, however again, the correction rendered them insignificant. 
Table 2 Physical parameters values at baseline and following 15 weeks of double-blind treatment

\begin{tabular}{|c|c|c|c|c|c|c|c|}
\hline & PS-DHA & & & Placebo & & & \\
\hline Variable & $\begin{array}{c}\text { Baseline } \\
\text { (mean } \pm \text { SD) }\end{array}$ & $\begin{array}{c}15 \text { weeks } \\
\text { (mean } \pm \text { SD) }\end{array}$ & $\begin{array}{l}\text { P value (within } \\
\text { group) }\end{array}$ & $\begin{array}{c}\text { Baseline } \\
(\text { mean } \pm \mathrm{SD})\end{array}$ & $\begin{array}{c}15 \text { weeks } \\
\text { (mean } \pm \text { SD) }\end{array}$ & $\begin{array}{l}\text { P value' (within } \\
\text { group) }\end{array}$ & $\begin{array}{l}\text { P value }{ }^{2} \text { (between } \\
\text { groups) }\end{array}$ \\
\hline $\begin{array}{l}\text { Resting systolic } \\
\text { BP }(\mathrm{mm} \mathrm{Hg})\end{array}$ & $127.1 \pm 13.4$ & $128.4 \pm 15.4$ & 0.511 & $127.6 \pm 15.8$ & $127.8 \pm 16.5$ & 0.909 & 0.685 \\
\hline Male & $125.4 \pm 13.5$ & $126.8 \pm 14.6$ & 0.612 & $129.0 \pm 13.0$ & $131.7 \pm 12.6$ & 0.244 & 0.691 \\
\hline Female & $128.8 \pm 13.3$ & $130.0 \pm 16.2$ & 0.676 & $126.1 \pm 18.7$ & $123.3 \pm 19.5$ & 0.330 & 0.331 \\
\hline $\begin{array}{l}\text { Resting diastolic } \\
\text { BP }(\mathrm{mm} \mathrm{Hg})\end{array}$ & $73.8 \pm 9.2$ & $72.6 \pm 9.1$ & 0.299 & $75.0 \pm 7.9$ & $73.3 \pm 9.4$ & 0.159 & 0.746 \\
\hline Male & $73.9 \pm 9.3$ & $71.7 \pm 9.0$ & 0.188 & $75.4 \pm 8.0$ & $73.9 \pm 9.7$ & 0.289 & 0.795 \\
\hline Female & $73.6 \pm 9.2$ & $73.4 \pm 9.3$ & 0.893 & $74.6 \pm 7.8$ & $72.7 \pm 9.2$ & 0.357 & 0.516 \\
\hline $\begin{array}{l}\text { Resting pulse } \\
\text { (beats/minute) }\end{array}$ & $69.4 \pm 10.1$ & $69.9 \pm 9.5$ & 0.657 & $66.5 \pm 8.4$ & $67.6 \pm 10.1$ & 0.394 & 0.725 \\
\hline Male & $69.1 \pm 11.8$ & $69.8 \pm 10.8$ & 0.648 & $63.9 \pm 7.8$ & $66.1 \pm 10.1$ & 0.139 & 0.482 \\
\hline Female & $69.7 \pm 8.4$ & $69.9 \pm 8.2$ & 0.872 & $69.6 \pm 8.2$ & $69.3 \pm 9.6$ & 0.865 & 0.812 \\
\hline Weight (kg) & $70.3 \pm 11.2$ & $70.8 \pm 11.6$ & $0.033^{*}$ & $73.0 \pm 12.9$ & $73.3 \pm 13.3$ & 0.209 & 0.622 \\
\hline Male & $77.4 \pm 9.6$ & $78.0 \pm 9.9$ & $0.036^{*}$ & $79.1 \pm 11.1$ & $79.5 \pm 11.5$ & 0.235 & 0.664 \\
\hline Female & $63.7 \pm 8.3$ & $64.0 \pm 8.7$ & 0.359 & $66.1 \pm 11.4$ & $66.3 \pm 11.9$ & 0.640 & 0.746 \\
\hline
\end{tabular}

${ }^{1}$ Based on two-sided $t$ test for dependent samples. ${ }^{2}$ Based on two-sided $t$ test for independent samples.

* Statistical significance was not maintained following Bonferonni correction.

In the treatment arm, 32 males and 33 females had blood pressure and pulse measurements at baseline and endpoint and 31 males and 33 females had weight measurements at baseline and endpoint. In the placebo arm, 34 males and 29 females had blood pressure and pulse measurements at baseline and endpoint and 33 males and 29 females had weight measurements at baseline and endpoint.

\section{Adverse Events}

Adverse events reported during the course of the double-blind phase are presented in Table 6. Twenty participants from the PS-DHA and 11 participants from the placebo group reported adverse events (29 and 15 adverse events, respectively). There were no significant differences between the study groups in the number of participants who reported an adverse event $(P=0.078)$ or in the number of reported adverse events $(\mathrm{P}=0.087)$. Ten participants from the PS-DHA group and 8 participants from the placebo group were classified by the study physicians as suffering from related or probably related adverse events (16 and 11 adverse events, respectively). Again, there were no significant differences between the study groups in the number of participants who reported an adverse event $(P=0.637)$ or in the number of reported adverse events, classified as related or probably related to the study treatment $(\mathrm{P}=0.472)$.

During the course of the double-blind phase, there were 5 severe adverse events (suspected acute diverticulitis, benign prostatic hyperplasia surgery, and 3 hospitalizations due to hyponatremia, bradycardia and abdominal

Table 3 Physical parameters values of PS-DHA continuers* at baseline and following 30 weeks of treatment

\begin{tabular}{lccc}
\hline Variable & Baseline $($ mean \pm SD) & 30 weeks $($ mean \pm SD) & P value \\
\hline Resting systolic BP $(\mathrm{mm} \mathrm{Hg})$ & $127.2 \pm 13.5$ & $126.3 \pm 14.0$ & 0.594 \\
\hline Male & $125.7 \pm 13.7$ & $126.5 \pm 14.3$ & 0.716 \\
\hline Female & $128.6 \pm 13.4$ & $126.0 \pm 14.0$ & 0.366 \\
\hline Resting diastolic BP $(\mathrm{mm} \mathrm{Hg})$ & $73.8 \pm 9.4$ & $70.7 \pm 9.8$ & 0.006 \\
\hline Male & $73.8 \pm 9.7$ & $71.6 \pm 8.7$ & 0.195 \\
\hline Female & $73.8 \pm 9.3$ & $69.9 \pm 10.7$ & 0.010 \\
\hline Resting pulse (beats/minute) & $69.4 \pm 10.0$ & $70.6 \pm 11.9$ & 0.368 \\
\hline Male & $69.3 \pm 11.6$ & $71.1 \pm 13.4$ & 0.412 \\
\hline Female & $69.5 \pm 8.5$ & $70.1 \pm 10.6$ & 0.687 \\
\hline Weight $(\mathrm{kg})$ & $70.0 \pm 11.2$ & $70.7 \pm 11.4$ & 0.015 \\
\hline Male & $77.1 \pm 9.6$ & $77.7 \pm 9.8$ & 0.067 \\
\hline Female & $63.7 \pm 8.5$ & $64.3 \pm 8.8$ & 0.104 \\
\hline
\end{tabular}

* include 29 males and 32 females.

$P$ value based on two-sided t test for dependent samples. 
Table 4 Biochemical parameters values at baseline and following 15 weeks of double-blind treatment

\begin{tabular}{|c|c|c|c|c|c|c|c|}
\hline \multirow[b]{2}{*}{ Variable } & \multicolumn{3}{|c|}{ PS-DHA } & \multicolumn{4}{|c|}{ Placebo } \\
\hline & $\begin{array}{c}\text { Baseline } \\
\text { (mean } \pm \text { SD) }\end{array}$ & $\begin{array}{c}15 \text { weeks } \\
\text { (mean } \pm \text { SD) }\end{array}$ & $\begin{array}{l}\text { P value }{ }^{1} \text { (within } \\
\text { group) }\end{array}$ & $\begin{array}{c}\text { Baseline } \\
\text { (mean } \pm \text { SD) }\end{array}$ & $\begin{array}{c}15 \text { weeks } \\
\text { (mean } \pm \text { SD) }\end{array}$ & $\begin{array}{l}\text { P value (within } \\
\text { group) }\end{array}$ & $\begin{array}{l}\text { P value (between } \\
\text { groups) }\end{array}$ \\
\hline Glucose $(\mathrm{mg} / \mathrm{dL})$ & $106.6 \pm 34.0$ & $105.9 \pm 37.1$ & 0.885 & $103.4 \pm 30.7$ & $111.1 \pm 47.3$ & 0.082 & 0.175 \\
\hline Male & $115.1 \pm 42.0$ & $110.4 \pm 47.4$ & 0.551 & $105.8 \pm 33.6$ & $118.3 \pm 59.9$ & 0.076 & 0.101 \\
\hline Female & $98.0 \pm 20.7$ & $101.5 \pm 22.5$ & 0.330 & $100.6 \pm 27.3$ & $102.9 \pm 25.6$ & 0.662 & 0.847 \\
\hline Sodium (mmol/L) & $140.6 \pm 2.6$ & $140.9 \pm 2.7$ & 0.316 & $140.9 \pm 2.1$ & $140.3 \pm 2.3$ & $0.014^{*}$ & $0.017^{*}$ \\
\hline Male & $140.6 \pm 2.6$ & $140.9 \pm 2.7$ & 0.352 & $141.2 \pm 2.0$ & $140.6 \pm 2.1$ & 0.092 & 0.061 \\
\hline Female & $140.5 \pm 2.7$ & $140.8 \pm 2.7$ & 0.582 & $140.6 \pm 2.2$ & $140.0 \pm 2.5$ & 0.079 & 0.136 \\
\hline Calcium (mg/dL) & $9.6 \pm 0.6$ & $9.6 \pm 0.5$ & 0.452 & $9.4 \pm 0.6$ & $9.6 \pm 0.5$ & $0.012^{*}$ & $0.038^{*}$ \\
\hline Male & $9.5 \pm 0.4$ & $9.5 \pm 0.5$ & 0.966 & $9.4 \pm 0.3$ & $9.5 \pm 0.4$ & 0.189 & 0.413 \\
\hline Female & $9.8 \pm 0.7$ & $9.6 \pm 0.5$ & 0.371 & $9.5 \pm 0.8$ & $9.7 \pm 0.5$ & $0.032^{*}$ & 0.051 \\
\hline $\begin{array}{l}\text { Phosphorus (mg/ } \\
\mathrm{dL} \text { ) }\end{array}$ & $3.4 \pm 0.6$ & $3.3 \pm 0.5$ & 0.103 & $3.4 \pm 0.5$ & $3.3 \pm 0.5$ & 0.346 & 0.807 \\
\hline Male & $3.2 \pm 0.6$ & $3.1 \pm 0.5$ & 0.190 & $3.2 \pm 0.5$ & $3.2 \pm 0.5$ & 0.636 & 0.671 \\
\hline Female & $3.7 \pm 0.5$ & $3.6 \pm 0.5$ & 0.326 & $3.5 \pm 0.6$ & $3.4 \pm 0.5$ & 0.397 & 0.932 \\
\hline Chloride $(\mathrm{mmol} / \mathrm{L})$ & $101.6 \pm 3.1$ & $102.4 \pm 3.0$ & $0.010^{*}$ & $101.9 \pm 3.1$ & $101.8 \pm 2.3$ & 0.880 & $0.047^{*}$ \\
\hline Male & $101.9 \pm 2.8$ & $102.6 \pm 3.0$ & 0.094 & $102.1 \pm 3.0$ & $102.1 \pm 2.2$ & 1.000 & 0.262 \\
\hline Female & $101.2 \pm 3.4$ & $102.2 \pm 2.9$ & 0.053 & $101.6 \pm 3.4$ & $101.5 \pm 2.4$ & 0.819 & 0.104 \\
\hline Potassium (mmol/L) & $4.6 \pm 0.5$ & $4.5 \pm 0.3$ & $0.031^{*}$ & $4.6 \pm 0.5$ & $4.7 \pm 0.7$ & 0.703 & 0.128 \\
\hline Male & $4.8 \pm 0.7$ & $4.6 \pm 0.4$ & 0.095 & $4.7 \pm 0.4$ & $4.7 \pm 0.6$ & 0.977 & 0.221 \\
\hline Female & $4.5 \pm 0.3$ & $4.4 \pm 0.3$ & 0.173 & $4.6 \pm 0.6$ & $4.6 \pm 0.8$ & 0.638 & 0.345 \\
\hline BUN (mg/dL) & $18.5 \pm 4.9$ & $18.9 \pm 4.8$ & 0.321 & $18.6 \pm 5.2$ & $19.7 \pm 6.7$ & $0.039^{*}$ & 0.262 \\
\hline Male & $19.6 \pm 4.8$ & $19.9 \pm 4.9$ & 0.679 & $20.5 \pm 5.3$ & $21.7 \pm 7.2$ & 0.101 & 0.249 \\
\hline Female ${ }^{* *}$ & $17.4 \pm 4.9$ & $18.0 \pm 4.7$ & 0.345 & $16.3 \pm 4.1$ & $17.3 \pm 5.3$ & 0.225 & 0.680 \\
\hline Creatinine (mg/dL) & $0.9 \pm 0.2$ & $0.9 \pm 0.2$ & 0.223 & $0.9 \pm 0.2$ & $0.9 \pm 0.2$ & 0.416 & 0.953 \\
\hline Male & $1.0 \pm 0.2$ & $1.0 \pm 0.2$ & 0.507 & $1.0 \pm 0.3$ & $1.0 \pm 0.3$ & 0.617 & 0.420 \\
\hline Female & $0.8 \pm 0.1$ & $0.8 \pm 0.2$ & 0.237 & $0.7 \pm 0.2$ & $0.8 \pm 0.2$ & $0.041^{*}$ & 0.265 \\
\hline $\begin{array}{l}\text { Alkaline } \\
\text { Phosphatase (U/L) }\end{array}$ & $68.3 \pm 22.5$ & $67.3 \pm 22.6$ & 0.331 & $69.9 \pm 30.0$ & $69.4 \pm 27.8$ & 0.578 & 0.726 \\
\hline Male & $69.6 \pm 24.9$ & $68.0 \pm 25.7$ & 0.380 & $73.3 \pm 36.2$ & $72.1 \pm 33.0$ & 0.286 & 0.842 \\
\hline Female & $67.1 \pm 20.1$ & $66.7 \pm 19.4$ & 0.693 & $66.0 \pm 20.8$ & $66.3 \pm 20.3$ & 0.862 & 0.724 \\
\hline ALT/SGPT (U/L) & $23.9 \pm 26.4$ & $20.7 \pm 8.4$ & 0.282 & $19.3 \pm 6.4$ & $19.5 \pm 5.8$ & 0.733 & 0.262 \\
\hline Male & $28.9 \pm 36.3$ & $22.3 \pm 9.7$ & 0.273 & $19.2 \pm 5.9$ & $19.2 \pm 5.8$ & 1.000 & 0.276 \\
\hline Female & $18.9 \pm 6.8$ & $19.0 \pm 6.6$ & 0.869 & $19.5 \pm 7.1$ & $19.9 \pm 5.8$ & 0.655 & 0.794 \\
\hline AST/SGOT (U/L) & $25.2 \pm 20.8$ & $22.4 \pm 6.1$ & 0.250 & $21.9 \pm 6.2$ & $21.8 \pm 5.3$ & 0.879 & 0.280 \\
\hline Male & $28.6 \pm 28.6$ & $23.3 \pm 7.1$ & 0.276 & $21.8 \pm 5.7$ & $21.3 \pm 5.5$ & 0.536 & 0.329 \\
\hline Female & $21.9 \pm 6.5$ & $21.5 \pm 5.0$ & 0.685 & $21.9 \pm 6.8$ & $22.3 \pm 5.0$ & 0.704 & 0.577 \\
\hline $\begin{array}{l}\text { Total Bilirubin }(\mathrm{mg} / \\
\mathrm{dL})\end{array}$ & $0.6 \pm 0.2$ & $0.6 \pm 0.3$ & 0.361 & $0.6 \pm 0.3$ & $0.6 \pm 0.3$ & 0.673 & 0.747 \\
\hline Male & $0.6 \pm 0.3$ & $0.6 \pm 0.3$ & 0.097 & $0.7 \pm 0.3$ & $0.7 \pm 0.3$ & 0.325 & 0.753 \\
\hline Female & $0.5 \pm 0.2$ & $0.5 \pm 0.2$ & 0.699 & $0.5 \pm 0.2$ & $0.5 \pm 0.2$ & 0.423 & 0.826 \\
\hline Total Protein $(\mathrm{g} / \mathrm{dL})$ & $7.3 \pm 0.4$ & $7.3 \pm 0.4$ & 0.190 & $7.3 \pm 0.5$ & $7.4 \pm 0.5$ & 0.188 & 0.936 \\
\hline Male & $7.3 \pm 0.4$ & $7.3 \pm 0.5$ & 0.358 & $7.3 \pm 0.4$ & $7.3 \pm 0.5$ & 0.990 & 0.489 \\
\hline Female & $7.3 \pm 0.4$ & $7.3 \pm 0.4$ & 0.362 & $7.4 \pm 0.6$ & $7.5 \pm 0.4$ & 0.091 & 0.443 \\
\hline $\begin{array}{l}\text { Triglycerides (mg/ } \\
\mathrm{dL} \text { ) }\end{array}$ & $141.6 \pm 61.4$ & $133.0 \pm 70.0$ & 0.133 & $128.9 \pm 54.8$ & $131.6 \pm 58.5$ & 0.677 & 0.186 \\
\hline Male & $138.2 \pm 68.1$ & $133.8 \pm 79.6$ & 0.577 & $131.6 \pm 54.9$ & $121.7 \pm 42.2$ & 0.198 & 0.611 \\
\hline Female & $145.1 \pm 54.8$ & $132.2 \pm 60.0$ & 0.132 & $125.9 \pm 55.4$ & $142.8 \pm 71.9$ & 0.103 & $0.025^{*}$ \\
\hline
\end{tabular}


Table 4 Biochemical parameters values at baseline and following 15 weeks of double-blind treatment (Continued)

\begin{tabular}{lccccccc}
\hline HDL $(\mathrm{mg} / \mathrm{dL})$ & $54.8 \pm 12.4$ & $56.4 \pm 13.4$ & 0.118 & $53.7 \pm 14.1$ & $54.4 \pm 14.1$ & 0.430 & 0.505 \\
\hline Male & $48.8 \pm 9.6$ & $50.4 \pm 10.5$ & $0.044^{*}$ & $48.3 \pm 10.2$ & $49.2 \pm 11.2$ & 0.348 & 0.604 \\
\hline Female & $60.9 \pm 12.1$ & $62.4 \pm 13.4$ & 0.404 & $59.9 \pm 15.4$ & $60.3 \pm 14.9$ & 0.785 & 0.635 \\
\hline LDL $(\mathrm{mg} / \mathrm{dL})$ & $97.6 \pm 31.4$ & $98.6 \pm 31.7$ & 0.745 & $98.9 \pm 30.9$ & $99.1 \pm 25.6$ & 0.940 & 0.853 \\
\hline Male & $88.6 \pm 30.4$ & $91.0 \pm 31.8$ & 0.287 & $94.4 \pm 28.9$ & $92.9 \pm 21.2$ & 0.656 & 0.332 \\
\hline Female & $106.6 \pm 30.2$ & $106.2 \pm 30.2$ & 0.946 & $104.1 \pm 32.8$ & $106.2 \pm 28.5$ & 0.672 & 0.743 \\
\hline $\begin{array}{l}\text { Total cholesterol } \\
\text { (mg/dL) }\end{array}$ & $177.5 \pm 38.2$ & $181.1 \pm 35.2$ & 0.402 & $178.0 \pm 37.2$ & $179.4 \pm 33.7$ & 0.650 & 0.687 \\
\hline Male & $164.6 \pm 31.2$ & $167.6 \pm 33.1$ & 0.283 & $168.5 \pm 33.2$ & $166.0 \pm 26.3$ & 0.487 & 0.226 \\
\hline Female & $190.3 \pm 40.6$ & $194.5 \pm 32.4$ & 0.613 & $188.7 \pm 39.0$ & $194.6 \pm 35.1$ & 0.284 & 0.856 \\
\hline
\end{tabular}

${ }^{1}$ Based on two-sided $t$ test for dependent samples. ${ }^{2}$ Based on two-sided $t$ test for independent samples

* Statistical significance was not maintained following Bonferonni correction

In the treatment arm, 33 males and 33 females had biochemical blood measurements at baseline and endpoint.

In the placebo arm, 34 males and 30 females had biochemical blood measurements at baseline and endpoint.

** In the placebo arm 29 females had BUN measurements at baseline and endpoint.

Table 5 Hematological parameters values at baseline and following 15 weeks of double-blind treatment

\begin{tabular}{|c|c|c|c|c|c|c|c|}
\hline & & PS-DHA & & & & Placebo & \\
\hline Variable & $\begin{array}{l}\text { Baseline (mean } \\
\pm \mathrm{SD})\end{array}$ & $\begin{array}{c}15 \text { weeks } \\
\text { (mean } \pm \\
\text { SD) }\end{array}$ & $\begin{array}{c}\text { P value (within } \\
\text { group) }\end{array}$ & $\begin{array}{c}\text { Baseline (mean } \\
\pm \mathrm{SD})\end{array}$ & $\begin{array}{c}15 \text { weeks } \\
\text { (mean } \pm \\
\text { SD) }\end{array}$ & $\begin{array}{l}\text { P value (within } \\
\text { group) }\end{array}$ & $\begin{array}{c}\text { P value }{ }^{2} \text { (between } \\
\text { groups) }\end{array}$ \\
\hline $\begin{array}{l}\text { White blood cell count } \\
\qquad\left(\times 10^{3} / \mathrm{cmm}\right)\end{array}$ & $6.8 \pm 1.8$ & $6.5 \pm 1.6$ & 0.083 & $7.1 \pm 2.0$ & $6.7 \pm 1.9$ & $0.011^{*}$ & 0.434 \\
\hline Male & $6.8 \pm 1.7$ & $6.4 \pm 1.5$ & $0.020^{*}$ & $7.3 \pm 2.3$ & $7.1 \pm 2.2$ & 0.298 & 0.467 \\
\hline Female & $6.7 \pm 1.9$ & $6.7 \pm 1.6$ & 0.829 & $6.9 \pm 1.4$ & $6.3 \pm 1.3$ & $0.009^{*}$ & 0.066 \\
\hline $\begin{array}{c}\text { Red blood cell count }\left(\times 10^{6} /\right. \\
\mathrm{cmm})\end{array}$ & $4.6 \pm 0.5$ & $4.6 \pm 0.5$ & 0.441 & $4.6 \pm 0.4$ & $4.6 \pm 0.4$ & 0.332 & 0.765 \\
\hline Male & $4.7 \pm 0.5$ & $4.7 \pm 0.5$ & 0.544 & $4.6 \pm 0.4$ & $4.6 \pm 0.4$ & 0.491 & 0.925 \\
\hline Female & $4.5 \pm 0.4$ & $4.4 \pm 0.4$ & 0.622 & $4.5 \pm 0.4$ & $4.5 \pm 0.4$ & 0.488 & 0.760 \\
\hline Hematocrit (\%) & $41.2 \pm 4.2$ & $\begin{array}{c}40.7 \pm \\
4.3\end{array}$ & 0.113 & $41.6 \pm 3.6$ & $\begin{array}{c}40.9 \pm \\
3.6\end{array}$ & $0.045^{*}$ & 0.504 \\
\hline Male & $42.5 \pm 4.3$ & $\begin{array}{c}42.1 \pm \\
4.4\end{array}$ & 0.229 & $42.6 \pm 3.6$ & $\begin{array}{c}42.0 \pm \\
3.4\end{array}$ & 0.100 & 0.763 \\
\hline Female & $39.8 \pm 3.7$ & $\begin{array}{c}39.4 \pm \\
3.8\end{array}$ & 0.309 & $40.5 \pm 3.2$ & $\begin{array}{c}39.6 \pm \\
3.5\end{array}$ & 0.193 & 0.548 \\
\hline Hemoglobin $(\mathrm{g} / \mathrm{dL})$ & $13.7 \pm 1.5$ & $\begin{array}{l}13.7 \pm \\
1.5\end{array}$ & 0.434 & $13.7 \pm 1.2$ & $\begin{array}{c}13.6 \pm \\
1.2\end{array}$ & 0.213 & 0.669 \\
\hline Male & $14.3 \pm 1.4$ & $\begin{array}{c}14.3 \pm \\
1.3\end{array}$ & 0.706 & $14.1 \pm 1.2$ & $\begin{array}{c}13.9 \pm \\
1.2\end{array}$ & 0.181 & 0.623 \\
\hline Female & $13.1 \pm 1.4$ & $\begin{array}{c}13.0 \pm \\
1.4\end{array}$ & 0.481 & $13.3 \pm 1.1$ & $\begin{array}{c}13.2 \pm \\
1.1\end{array}$ & 0.494 & 0.856 \\
\hline Platelet count $\left(\times 10^{3} / \mathrm{cmm}\right)$ & $221.5 \pm 62.7$ & $\begin{array}{c}226.8 \pm \\
60.5\end{array}$ & 0.374 & $226.9 \pm 73.7$ & $\begin{array}{c}230.8 \pm \\
68.8\end{array}$ & 0.347 & 0.839 \\
\hline Male & $200.1 \pm 44.5$ & $\begin{array}{c}204.0 \pm \\
44.3\end{array}$ & 0.479 & $202.8 \pm 58.3$ & $\begin{array}{c}210.0 \pm \\
61.1\end{array}$ & 0.128 & 0.648 \\
\hline Female & $243.5 \pm 71.3$ & $\begin{array}{c}250.2 \pm \\
66.4\end{array}$ & 0.536 & $255.4 \pm 80.6$ & $\begin{array}{l}255.3 \pm \\
70.4\end{array}$ & 0.980 & 0.592 \\
\hline Netrophils (\%) & $62.8 \pm 6.5$ & $\begin{array}{c}62.4 \pm \\
6.4\end{array}$ & 0.514 & $63.8 \pm 8.7$ & $\begin{array}{c}62.9 \pm \\
8.6\end{array}$ & 0.207 & 0.543 \\
\hline Male & $65.1 \pm 6.0$ & $\begin{array}{c}64.4 \pm \\
5.7\end{array}$ & 0.390 & $64.8 \pm 9.0$ & $\begin{array}{c}64.8 \pm \\
9.2\end{array}$ & 0.968 & 0.569 \\
\hline Female & $60.4 \pm 6.2$ & $\begin{array}{c}60.5 \pm \\
6.7\end{array}$ & 0.969 & $62.6 \pm 8.4$ & $\begin{array}{c}60.7 \pm \\
7.5\end{array}$ & 0.097 & 0.147 \\
\hline
\end{tabular}


Table 5 Hematological parameters values at baseline and following 15 weeks of double-blind treatment (Continued)

\begin{tabular}{|c|c|c|c|c|c|c|c|}
\hline Netrophils Absolute $\left(\times 10^{9} / \mathrm{L}\right)$ & $4.3 \pm 1.3$ & $4.1 \pm 1.0$ & 0.080 & $4.5 \pm 1.4$ & $4.2 \pm 1.4$ & $0.037^{*}$ & 0.579 \\
\hline Male & $4.5 \pm 1.3$ & $4.1 \pm 1.1$ & $0.027^{*}$ & $4.7 \pm 1.5$ & $4.6 \pm 1.7$ & 0.675 & 0.265 \\
\hline Female & $4.1 \pm 1.2$ & $4.0 \pm 1.0$ & 0.814 & $4.4 \pm 1.2$ & $3.8 \pm 1.0$ & $0.010^{*}$ & $0.046^{*}$ \\
\hline Lymphocytes (\%) & $26.0 \pm 6.1$ & $\begin{array}{c}26.1 \pm \\
5.8\end{array}$ & 0.887 & $25.8 \pm 8.7$ & $\begin{array}{c}26.4 \pm \\
8.1\end{array}$ & 0.324 & 0.478 \\
\hline Male & $23.8 \pm 5.6$ & $\begin{array}{c}24.4 \pm \\
5.1 \\
\end{array}$ & 0.453 & $24.5 \pm 8.8$ & $\begin{array}{c}24.4 \pm \\
8.7\end{array}$ & 0.893 & 0.529 \\
\hline Female & $28.2 \pm 5.8$ & $\begin{array}{c}27.8 \pm \\
6.0\end{array}$ & 0.447 & $27.3 \pm 8.4$ & $\begin{array}{c}28.8 \pm \\
6.9\end{array}$ & 0.171 & 0.116 \\
\hline $\begin{array}{c}\text { Lymphocytes Absolute }\left(\times 10^{9} /\right. \\
\text { L) }\end{array}$ & $1.8 \pm 0.6$ & $1.7 \pm 0.5$ & 0.167 & $1.9 \pm 1.1$ & $1.8 \pm 0.8$ & 0.051 & 0.449 \\
\hline Male & $1.6 \pm 0.5$ & $1.6 \pm 0.4$ & 0.234 & $1.9 \pm 1.4$ & $1.7 \pm 1.0$ & 0.115 & 0.468 \\
\hline Female & $1.9 \pm 0.6$ & $1.8 \pm 0.6$ & 0.458 & $1.8 \pm 0.6$ & $1.8 \pm 0.5$ & 0.238 & 0.819 \\
\hline Monocytes (\%) & $5.8 \pm 1.4$ & $6.0 \pm 1.5$ & 0.119 & $5.6 \pm 1.0$ & $5.9 \pm 1.4$ & 0.093 & 0.918 \\
\hline Male & $5.9 \pm 1.5$ & $6.1 \pm 1.4$ & 0.343 & $5.7 \pm 0.9$ & $5.9 \pm 1.3$ & 0.299 & 0.976 \\
\hline Female & $5.6 \pm 1.4$ & $5.9 \pm 1.6$ & 0.220 & $5.5 \pm 1.1$ & $5.8 \pm 1.5$ & 0.193 & 0.895 \\
\hline Monocytes Absolute $\left(\times 10^{9} / \mathrm{L}\right)$ & $0.4 \pm 0.1$ & $0.4 \pm 0.1$ & 0.731 & $0.4 \pm 0.1$ & $0.4 \pm 0.1$ & 0.565 & 0.516 \\
\hline Male & $0.4 \pm 0.1$ & $0.4 \pm 0.1$ & 0.497 & $0.4 \pm 0.1$ & $0.4 \pm 0.1$ & 0.890 & 0.556 \\
\hline Female & $0.4 \pm 0.1$ & $0.4 \pm 0.1$ & 0.284 & $0.4 \pm 0.1$ & $0.4 \pm 0.1$ & 0.359 & 0.159 \\
\hline Eosinophils (\%) & $3.0 \pm 2.5$ & $3.1 \pm 3.3$ & 0.365 & $2.5 \pm 1.6$ & $2.5 \pm 1.5$ & 0.844 & 0.540 \\
\hline Male & $2.7 \pm 1.5$ & $2.8 \pm 2.0$ & 0.535 & $2.8 \pm 1.8$ & $2.7 \pm 1.7$ & 0.832 & 0.533 \\
\hline Female & $3.3 \pm 3.3$ & $3.5 \pm 4.2$ & 0.513 & $2.2 \pm 1.4$ & $2.3 \pm 1.2$ & 0.660 & 0.796 \\
\hline Eosinophils Absolute $\left(\times 10^{9} / \mathrm{L}\right)$ & $0.2 \pm 0.3$ & $0.2 \pm 0.3$ & 0.853 & $0.2 \pm 0.1$ & $0.2 \pm 0.1$ & 0.227 & 0.400 \\
\hline Male & $0.2 \pm 0.2$ & $0.2 \pm 0.2$ & 0.421 & $0.2 \pm 0.1$ & $0.2 \pm 0.1$ & 0.280 & 0.943 \\
\hline Female & $0.2 \pm 0.4$ & $0.3 \pm 0.5$ & 0.382 & $0.1 \pm 0.1$ & $0.1 \pm 0.1$ & 0.513 & 0.284 \\
\hline Basophils (\%) & $0.7 \pm 0.3$ & $0.6 \pm 0.3$ & $0.041^{*}$ & $0.7 \pm 0.3$ & $0.6 \pm 0.3$ & 0.101 & 0.684 \\
\hline Male & $0.7 \pm 0.3$ & $0.6 \pm 0.3$ & 0.207 & $0.6 \pm 0.3$ & $0.6 \pm 0.3$ & 0.099 & 0.882 \\
\hline Female & $0.8 \pm 0.2$ & $0.6 \pm 0.3$ & 0.113 & $0.7 \pm 0.4$ & $0.7 \pm 0.3$ & 0.515 & 0.482 \\
\hline Basophils Absolute $\left(\times 10^{9} / \mathrm{L}\right)$ & $0.1 \pm 0.0$ & $0.0 \pm 0.0$ & $0.006^{*}$ & $0.1 \pm 0.0$ & $0.0 \pm 0.0$ & $0.019^{*}$ & 0.854 \\
\hline Male & $0.1 \pm 0.0$ & $0.0 \pm 0.0$ & $0.035^{*}$ & $0.1 \pm 0.0$ & $0.0 \pm 0.0$ & 0.058 & 0.928 \\
\hline Female & $0.1 \pm 0.0$ & $0.0 \pm 0.0$ & 0.077 & $0.1 \pm 0.0$ & $0.0 \pm 0.0$ & 0.174 & 0.701 \\
\hline Large Unstained Cells (\%) & $1.7 \pm 0.6$ & $1.7 \pm 0.5$ & 1.000 & $1.6 \pm 0.6$ & $1.7 \pm 0.6$ & 0.162 & 0.306 \\
\hline Male & $1.8 \pm 0.6$ & $1.8 \pm 0.5$ & 0.616 & $1.6 \pm 0.6$ & $1.6 \pm 0.6$ & 0.623 & 0.483 \\
\hline Female & $1.7 \pm 0.7$ & 1.7. \pm 0.6 & 0.701 & $1.7 \pm 0.5$ & $1.9 \pm 0.6$ & 0.139 & 0.434 \\
\hline $\begin{array}{l}\text { Large Unstained Cells } \\
\text { Absolute }\left(\times 10^{9} / \mathrm{L}\right)\end{array}$ & $0.1 \pm 0.0$ & $0.1 \pm 0.0$ & 0.652 & $0.1 \pm 0.1$ & $0.1 \pm 0.1$ & 0.571 & 0.948 \\
\hline Male & $0.1 \pm 0.0$ & $0.1 \pm 0.0$ & 0.243 & $0.1 \pm 0.1$ & $0.1 \pm 0.1$ & 0.568 & 0.753 \\
\hline Female & $0.1 \pm 0.1$ & $0.1 \pm 0.0$ & 0.784 & $0.1 \pm 0.0$ & $0.1 \pm 0.0$ & 0.847 & 0.742 \\
\hline $\operatorname{MCV}\left(\mu^{3}\right)$ & $90.1 \pm 4.1$ & $\begin{array}{c}89.6 \pm \\
4.4\end{array}$ & 0.133 & $91.0 \pm 4.3$ & $\begin{array}{c}90.1 \pm \\
4.3\end{array}$ & $0.049^{*}$ & 0.506 \\
\hline Male & $90.7 \pm 4.4$ & $\begin{array}{c}90.2 \pm \\
4.4\end{array}$ & 0.204 & $92.2 \pm 4.3$ & $\begin{array}{l}91.4 \pm \\
4.6\end{array}$ & 0.211 & 0.780 \\
\hline Female & $89.5 \pm 3.9$ & $\begin{array}{c}89.1 \pm \\
4.31\end{array}$ & 0.397 & $89.6 \pm 3.9$ & $\begin{array}{l}88.7 \pm \\
3.6\end{array}$ & 0.124 & 0.503 \\
\hline $\mathbf{M C H}(\mathrm{pg})$ & $30.1 \pm 2.1$ & $\begin{array}{c}30.1 \pm \\
1.9 \\
\end{array}$ & 0.850 & $30.0 \pm 1.5$ & $\begin{array}{c}30.0 \pm \\
1.4\end{array}$ & 0.940 & 0.861 \\
\hline Male & $30.6 \pm 2.2$ & $\begin{array}{l}30.7 \pm \\
2.0 \\
\end{array}$ & 0.651 & $30.4 \pm 1.6$ & $\begin{array}{l}30.4 \pm \\
1.5 \\
\end{array}$ & 0.842 & 0.658 \\
\hline Female & $29.5 \pm 1.8$ & $\begin{array}{c}29.5 \pm \\
1.8\end{array}$ & 0.801 & $29.5 \pm 1.1$ & $\begin{array}{c}29.5 \pm \\
1.1\end{array}$ & 0.910 & 0.810 \\
\hline
\end{tabular}


Table 5 Hematological parameters values at baseline and following 15 weeks of double-blind treatment (Continued)

\begin{tabular}{|c|c|c|c|c|c|c|c|}
\hline MCHC (\%) & $33.4 \pm 1.6$ & $\begin{array}{c}33.6 \pm \\
1.4\end{array}$ & 0.108 & $33.0 \pm 1.2$ & $\begin{array}{c}33.3 \pm \\
0.9\end{array}$ & $0.041^{*}$ & 0.673 \\
\hline Male & $33.8 \pm 1.9$ & $\begin{array}{c}34.1 \pm \\
1.7\end{array}$ & 0.116 & $33.0 \pm 1.2$ & $\begin{array}{c}33.2 \pm \\
1.1\end{array}$ & 0.267 & 0.794 \\
\hline Female & $32.9 \pm 1.1$ & $\begin{array}{c}33.1 \pm \\
0.9\end{array}$ & 0.533 & $32.9 \pm 1.1$ & $\begin{array}{c}33.3 \pm \\
0.7\end{array}$ & 0.061 & 0.335 \\
\hline
\end{tabular}

${ }^{1}$ Based on two-sided $t$ test for dependent samples. ${ }^{2}$ Based on two-sided $t$ test for independent samples

* Statistical significance was not maintained following Bonferonni correction

In the treatment arm, 33 males and 32 females had hematological blood measurements at baseline and endpoint.

In the placebo arm, 33 males and 28 females had hematological blood measurements at baseline and endpoint.

discomfort) in 5 participants in the PS-DHA group and 2 severe adverse events (atrial fibrillation and epigastric pain leading to hospitalization) in one participant in the placebo group. All the severe adverse events were classified by the study physicians as not related or probably not related to the study treatment.
Adverse events reported during the course of the open-label extension are presented in Table 7. Ten participants from the PS-DHA continuers reported 12 adverse events of which 3 were classified by the study physicians as related or probably related to the study treatment. Six participants from the PS-DHA naive

Table 6 Adverse events reported during the course of the double-blind phase*

\begin{tabular}{|c|c|c|c|c|}
\hline \multirow[b]{2}{*}{ Adverse event } & \multicolumn{2}{|c|}{ PS-DHA } & \multicolumn{2}{|c|}{ Placebo } \\
\hline & $\begin{array}{c}\text { Number of related or } \\
\text { probably related adverse } \\
\text { events }\end{array}$ & $\begin{array}{l}\text { Number of not-related or } \\
\text { probably not related } \\
\text { adverse events }\end{array}$ & $\begin{array}{c}\text { Number of related or } \\
\text { probably related adverse } \\
\text { events }\end{array}$ & $\begin{array}{l}\text { Number of not-related or } \\
\text { probably not related } \\
\text { adverse events }\end{array}$ \\
\hline \multicolumn{5}{|l|}{$\begin{array}{l}\text { Adverse events among } \\
\text { study drop-outs }\end{array}$} \\
\hline $\begin{array}{l}\text { Gastrointestinal } \\
\text { discomfort }\end{array}$ & 4 & & 1 & \\
\hline Rush & 1 & & & \\
\hline $\begin{array}{c}\text { Increased appetite and } \\
\text { weight }\end{array}$ & & & 1 & \\
\hline Strange general feeling & & & 1 & \\
\hline Headache & & & 2 & \\
\hline dizziness & & & 2 & \\
\hline Mood swings & & & 1 & \\
\hline SUM & 5 events in 5 participants & & 8 events in 5 participants & \\
\hline \multicolumn{5}{|l|}{$\begin{array}{l}\text { Adverse events among } \\
\text { study completers }\end{array}$} \\
\hline $\begin{array}{l}\text { Gastrointestinal } \\
\text { discomfort }\end{array}$ & 9 & 3 & 1 & 2 \\
\hline Weight loss & 1 & 1 & & \\
\hline Gastritis & & 1 & & \\
\hline Headache & 1 & 1 & & \\
\hline Pneumonia & & 2 & & \\
\hline Hypertension & & 2 & & 1 \\
\hline Hypothyroidism & & 1 & & \\
\hline Back pain & & 1 & & \\
\hline Leg wound & & 1 & & \\
\hline Redness in the mouth & & & 1 & \\
\hline Pruritus & & & 1 & \\
\hline Eyes inflammation & & & & 1 \\
\hline SUM & 11 events in 5 participants & 13 events in 10 participants & 3 events in 3 participants & 4 events in 3 participants \\
\hline
\end{tabular}

* Judged by the study physicians as related, probably related, not related or probably not related to the study treatment 
Table 7 Adverse events reported during the course of the open-label extension*

\begin{tabular}{|c|c|c|c|}
\hline \multicolumn{2}{|c|}{ PS-DHA continuers } & \multicolumn{2}{|c|}{ PS-DHA naive ${ }^{* *}$} \\
\hline Adverse event & $\begin{array}{l}\text { Number of related or probably } \\
\text { related adverse events }\end{array}$ & $\begin{array}{l}\text { Number of not-related or probably not } \\
\text { related adverse events }\end{array}$ & $\begin{array}{l}\text { Number of not-related or probably not } \\
\text { related adverse events }\end{array}$ \\
\hline $\begin{array}{l}\text { Gastrointestinal } \\
\text { discomfort }\end{array}$ & 1 & & 3 \\
\hline Hypertension & & 1 & 3 \\
\hline Hypotension & & 1 & \\
\hline Fall & & 1 & \\
\hline Tenesmus & 1 & & \\
\hline Amaurosis fugax & & 1 & \\
\hline $\begin{array}{l}\text { Mild bleeding due to } \\
\text { Hemorrhoids }\end{array}$ & & 1 & \\
\hline Platelets decrease & & 1 & \\
\hline Hand Fracture & & 1 & \\
\hline Hyperlipidemia & & & 1 \\
\hline Dizziness & & 1 & \\
\hline Urinal tract infection & & & 1 \\
\hline Arm pigmentation & & & 1 \\
\hline Headache & 1 & 1 & \\
\hline SUM & 3 events in 3 participants & 9 events in 7 participants & 9 events in 6 participants \\
\hline
\end{tabular}

group reported 9 adverse events; none of which were classified as related or probably related to the study treatment.

During the course of this phase, there were 4 severe adverse events (elective hospitalization for computed tomography urography, and chest pains, ear cholesteotoma and prostate surgery, leading to hospitalization), all classified by the study physicians as not related or probably not related to the study treatment.

\section{Discussion}

PS is widely used as an over the counter (OTC) preparation in the aim of improving general health and particularly cognitive functions of elderly people. Traditionally, PS has been extracted from bovine brain; however, recently, the BSE epidemic necessitated the finding of alternative safe sources such as SB-PS. SB-PS, however, differs considerably from BC-PS mainly in the absence of DHA which is the predominant omega-3 LC-PUFA in the mammalian central nervous system. Observational and epidemiological studies have associated omega-3 LCPUFA consumption with a reduced risk of impaired cognitive function in middle-aged population [19], and with a reduced risk of dementia $[20,21]$. This association has been supported by interventional studies [22-24].

To gain the benefits of mammalian PS without the attendant risks, a safe-sourced PS with omega-3 LC-PUFA attached to its backbone (PS- omega-3) was developed.
This compound was recently found to improve the symptoms of children with impaired visual sustained attention [15] and to protect middle-aged rats from scopolamineinduced deleterious effects [16]. The mechanism by which PS- omega-3 exerts its effects is not fully understood, however PS has been found to regulate key proteins in neuronal membranes, including sodium/calcium ATPase [25], protein kinase C [26] and Raf-1 protein kinase [27]. PS was also found to influence neurotransmitter activity, such as the release of acetylcholine, dopamine and noradrenaline [28]. In addition, PS- omega-3 was found to significantly increase DHA level in brains of middle-aged rats [16].

We have recently reported that a novel PS preparation (PS-DHA) may improve cognitive performance in nondemented elderly with memory complaints [17] and the safety results of this study are described herein.

Participants who discontinued the double-blind phase due to adverse events were distributed equally over the two study arms. No significant findings were observed in the tested physical, hematological or biochemical parameters following 15 weeks of treatment and there was no significant difference in the frequency of adverse events between the groups.

No subject discontinued the open-label phase due to an adverse event and there were no significant findings in the tested physical parameters.

Overall, the treatment was well tolerated with the most frequent symptom related to gastrointestinal 
discomfort. These symptoms were considered mild by the subjects and the physicians, and were previously reported for other PS compounds [29]. No severe adverse events were classified by the study physicians as related or probably related to the study treatment.

\section{Conclusions}

Taken in combination with the positive cognitive effects of the PS-DHA preparation reported previously [17], the study results support the safe use of PS-DHA for elderly people with memory complaints at a dosage of $300 \mathrm{mg}$ $\mathrm{PS} /$ day for 15 weeks, or at a lower dose (100 mg PS/day) for 30 weeks.

\section{Acknowledgements}

We thank Dr. Ruta Verchovsky and Dr. Ilan Halperin from the Neurology Department of Tel-Aviv Sourasky Medical Center for monitoring the participants' health and cognitive status throughout the study. We also thank Rachel Konopinsky-Link from the Neurology Department of Tel-Aviv Sourasky Medical Center for coordinating the study.

\section{Author details}

${ }^{1}$ Neurology Department, Tel-Aviv Sourasky Medical Center, Tel-Aviv, Israel. ${ }^{2}$ Enzymotec Ltd. P.O.Box 6, 23106, Migdal HaEmeq, Israel. ${ }^{3}$ Sieratzki Chair of Neurology, Tel-Aviv University Medical School, Ramat Aviv, Israel.

\section{Authors' contributions}

ADK, YR and $Y H$ interpreted the data and wrote the draft of this manuscript. The study protocol was designed by $W, A D K, Y R$ and TC. W and ADK were responsible for patient recruitment and management. All authors were involved in revising the manuscript and gave final approval for publication.

\section{Competing interests}

This study was funded by Enzymotec LTD, Israel. YR, TC and YH are employees of Enzymotec Ltd, and ADK served as consultant to the study.

Received: 13 February 2011 Accepted: 28 June 2011

Published: 28 June 2011

\section{References}

1. Vance JE, Steenbergen R: Metabolism and functions of phosphatidylserine. Prog Lipid Res 2005, 44(4):207-234.

2. Crook TH, Tinklenberg J, Yesavage J, Petrie W, Nunzi MG, Massari DC: Effects of phosphatidylserine in age-associated memory impairment. Neurology 1991, 41(5):644-649.

3. Cenacchi T, Bertoldin T, Farina C, Fiori MG, Crepaldi G: Cognitive decline in the elderly: a double-blind, placebo-controlled multicenter study on efficacy of phosphatidylserine administration. Aging (Milano) 1993, 5(2):123-133.

4. Amaducci L: Phosphatidylserine in the treatment of Alzheimer's disease: results of a multicenter study. Psychopharmacol Bull 1988, 24(1):130-134.

5. Crook T, Petrie W, Wells C, Massari DC: Effects of phosphatidylserine in Alzheimer's disease. Psychopharmacol Bull 1992, 28(1):61-66.

6. Palmieri G, Palmieri R, Inzoli MR, Lombardi G, Sottini C, Tavolato B, Giometto B: Double blind controlled trial of phosphatidylserine in patients with senile mental deterioration. Clinical Trials Journal 1987, 24(1):73-83.

7. Puca FM, Bavarese MA, Minervini MG: Exploratory trial of PS efficacy in mildly demented patients. Cinical Trials Journal 1987, 24:94-98.

8. Rabboni M, Maggioni FS, Giannelli A, Beinat L: Neuroendocrine and behavioral effects of PS in elderly patients with abiotrophic or vascular dementia or mild depression. Clinical Trials Journal 1990, 27:230-240.

9. Jager R, Purpura M, Kingsley M: Phospholipids and sports performance. $J$ Int Soc Sports Nutr 2007, 4:5.

10. Benton D, Donohoe RT, Sillance B, Nabb S: The influence of phosphatidylserine supplementation on mood and heart rate when faced with an acute stressor. Nutr Neurosci 2001, 4(3):169-178.
11. Hellhammer J, Fries E, Buss C, Engert V, Tuch A, Rutenberg D, Hellhammer D: Effects of soy lecithin phosphatidic acid and phosphatidylserine complex (PAS) on the endocrine and psychological responses to mental stress. Stress 2004, 7(2):119-126.

12. Jorissen BL, Brouns F, Van Boxtel MP, Ponds RW, Verhey FR, Jolles J, Riedel WJ: The influence of soy-derived phosphatidylserine on cognition in age-associated memory impairment. Nutr Neurosci 2001, 4(2):121-134

13. Schreiber S, Kampf-Sherf O, Gorfine M, Kelly D, Oppenheim Y, Lerer B: An open trial of plant-source derived phosphatydilserine for treatment of age-related cognitive decline. Isr J Psychiatry Relat Sci 2000, 37(4):302-307.

14. Jorissen BL, Brouns F, Van Boxtel MP, Riedel WJ: Safety of soy-derived phosphatidylserine in elderly people. Nutr Neurosci 2002, 5(5):337-343.

15. Vaisman N, Kaysar N, Zaruk-Adasha Y, Pelled D, Brichon G, Zwingelstein G, Bodennec J: Correlation between changes in blood fatty acid composition and visual sustained attention performance in children with inattention: effect of dietary $n-3$ fatty acids containing phospholipids. Am J Clin Nutr 2008, 87(5):1170-1180.

16. Vaisman N, Pelled D: n-3 phosphatidylserine attenuated scopolamineinduced amnesia in middle-aged rats. Prog Neuropsychopharmacol Biol Psychiatry 2009, 33(6):952-959.

17. Vakhapova V, Cohen T, Richter Y, Herzog Y, Korczyn AD: Phosphatidylserine Containing omega-3 Fatty Acids May Improve Memory Abilities in NonDemented Elderly with Memory Complaints: A Double-Blind PlaceboControlled Trial. Dement Geriatr Cogn Disord 29(5):467-474.

18. Crook TH, Feher EP, Larrabee GJ: Assessment of memory complaint in age-associated memory impairment: the MAC-Q. Int Psychogeriatr 1992, 4(2):165-176.

19. Kalmijn S, van Boxtel MP, Ocke M, Verschuren WM, Kromhout D, Launer LJ: Dietary intake of fatty acids and fish in relation to cognitive performance at middle age. Neurology 2004, 62(2):275-280.

20. Albanese E, Dangour AD, Uauy R, Acosta D, Guerra M, Guerra SSG, Huang Y, Jacob KS, Llibre de Rodriguez J, Noriega LH, et al: Dietary fish and meat intake and dementia in Latin America, China, and India: a 10/66 Dementia Research Group population-based study. Am J Clin Nutr 2009, 90(2):392-400.

21. Huang $T L$, Zandi PP, Tucker $K L$, Fitzpatrick $A L$, Kuller LH, Fried LP, Burke $G L$, Carlson MC: Benefits of fatty fish on dementia risk are stronger for those without APOE epsilon4. Neurology 2005, 65(9):1409-1414.

22. Fontani G, Corradeschi F, Felici A, Alfatti F, Migliorini S, Lodi L: Cognitive and physiological effects of Omega-3 polyunsaturated fatty acid supplementation in healthy subjects. Eur J Clin Investigation 2005, 35:691-699.

23. Terano T, Fujishiro S, Ban T, Yamamoto K, Tanaka T, Noguchi Y, Tamura Y, Yazawa $K$, Hirayama T: Docosahexaenoic acid supplementation improves the moderately severe dementia from thrombotic cerebrovascular diseases. Lipids 1999, 34(Suppl):S345-346.

24. Yurko-Mauro K, McCarthy D, Rom D, Nelson EB, Ryan AS, Blackwell A, Salem N $\mathrm{Jr}$, Stedman M: Beneficial effects of docosahexaenoic acid on cognition in age-related cognitive decline. Alzheimers Dement 2010, 6(6):456-464.

25. Wheeler KP, Whittam R: ATPase activity of the sodium pump needs phosphatidylserine. Nature 1970, 225(5231):449-450.

26. Bittova L, Stahelin RV, Cho W: Roles of ionic residues of the $\mathrm{C} 1$ domain in protein kinase $\mathrm{C}$-alpha activation and the origin of phosphatidylserine specificity. J Biol Chem 2001, 276(6):4218-4226.

27. Vance JE: Phosphatidylserine and phosphatidylethanolamine in mammalian cells: two metabolically related aminophospholipids. J Lipid Res 2008, 49(7):1377-1387.

28. Pepeu G, Pepeu IM, Amaducci L: A review of phosphatidylserine pharmacological and clinical effects. Is phosphatidylserine a drug for the ageing brain? Pharmacol Res 1996, 33(2):73-80.

29. Pepping J: Phosphatidylserine. American Journal of Health-System Pharmacy 1999, 56:2043-2044.

Pre-publication history

The pre-publication history for this paper can be accessed here: http://www.biomedcentral.com/1471-2377/11/79/prepub

doi:10.1186/1471-2377-11-79

Cite this article as: Vakhapova et al: Safety of phosphatidylserine containing omega-3 fatty acids in non-demented elderly: a doubleblind placebo-controlled trial followed by an open-label extension. BMC Neurology 2011 11:79. 